Trans

continentales
Transcontinentales

Sociétés, idéologies, système mondial

$10 / 11 \mid 2011$

La ruée vers la terre

\title{
Pratiques de l'appropriation foncière en contexte musulman
}

Land appropriation practices in the Muslim context

Yazid Ben Hounet, Barbara Casciarri, Baudouin Dupret, François Ireton et Alice Wilson

\section{OpenEdition}

1 Journals

Édition électronique

URL : http://journals.openedition.org/transcontinentales/1275

ISBN : 978-2-7351-1572-3

ISSN : 1775-397X

Éditeur

Editions de la maison des sciences de l'homme

Référence électronique

Yazid Ben Hounet, Barbara Casciarri, Baudouin Dupret, François Ireton et Alice Wilson, «Pratiques de I'appropriation foncière en contexte musulman », Transcontinentales [En ligne], 10/11 | 2011, document 7, mis en ligne le 19 octobre 2011, consulté le 07 septembre 2020. URL : http:// journals.openedition.org/transcontinentales/1275

Ce document a été généré automatiquement le 7 septembre 2020

Tous droits réservés 


\title{
Pratiques de l'appropriation foncière en contexte musulman
}

\author{
Land appropriation practices in the Muslim context \\ Yazid Ben Hounet, Barbara Casciarri, Baudouin Dupret, François Ireton et \\ Alice Wilson
}

\section{Introduction}

Dans les mondes musulmans, l'étude du droit et des institutions judiciaires a été largement négligée à l'exception précisément de ce qui touche à la référence à l'islam. Pour pallier ce défaut, nous proposons de substituer à l'anthropologie du droit musulman une anthropologie du droit dans les mondes musulmans. Nous présenterons donc ici des pistes pour une ethnographie comparée des pratiques liées à la propriété foncière et à sa transmission dans des contextes musulmans, en particulier soudanais et algérien ${ }^{1}$. Cela nous donnera les moyens empiriques de documenter la conviction que le droit n'est pas tant l'expression d'une culture qu'un ensemble de procédés, reconnus comme tels, visant à la stabilisation des relations humaines et donc, le cas échéant, à la solution des litiges qu'elles peuvent générer.

Loin de toute perspective tenant le droit pour le miroir de la société, nous pensons utile de décrire comment le droit s'accomplit dans un rapport actif à des règles travaillées par les usagers, et de traiter de la question du droit à partir des pratiques, du langage et des textes. En outre, nous cherchons à montrer que la référence à l'autorité de l'islam est occasionnelle et s'inscrit dans la banalité et la routine de l'accomplissement pratique de l'activité juridique.

3 Les pratiques liées à des questions de propriété foncière et de transmission de celle-ci peuvent relever ou non d'un État, de même que les droits de référence. Il peut donc y avoir des situations plurales et les deux contextes soudanais et algérien nous permettront d'en rendre compte. En même temps, il convient de ne pas assimiler l'immense variété des pratiques juridiques avec l'idée d'une pluralité des droits. L'approche praxéologique que nous suggérons porte sur ces pratiques qui se 
développent autour d'un objet de référence identifié par les acteurs comme étant du droit (qu'il s'agisse d'un droit étatique ou d'un autre droit reconnu comme tel), que ce soit pour l'interpréter, le mettre en œuvre, le contourner, le vider de toute substance ou le contester.

La question de la propriété et de sa transmission est au cœur même de toute interrogation socio-anthropologique. Elle est au principe des relations économiques et politiques fondant toute société, et ces relations constituent l'objet central de la régulation juridique. S'agissant des contextes soudanais et algérien, on n'insistera jamais assez sur le fait que, dans les sociétés postcoloniales, la transformation de l'occupation foncière en accès à sa propriété a représenté un des enjeux majeurs, bien que parfois occulté derrière diverses rhétoriques, des premières décennies de l'indépendance.

5 L'intérêt de se fixer sur le conflit et son règlement ne doit pas procéder d'une volonté de trouver une solution au problème définitionnel du droit, mais d'un souci de cadrage de l'étude comparative que cette contribution propose. Le conflit et son règlement peuvent nous servir de révélateurs des normes de référence et des méthodes de référencement, ainsi que du contexte dans lequel ce conflit s'inscrit. L'analyse d'un conflit portant sur une question de propriété peut alors rendre explicites les règles de la propriété en vigueur dans un contexte donné, les différentes façons que les gens concernés ont de s'y référer, la forme qu'un différend peut avoir dès lors qu'il est saisi par une instance chargée d'en traiter, mais aussi les relations du droit avec d'autres types de normativité et la structure, voire la nature, de ces autres normativités telles qu'elles sont perçues à partir des lieux du droit.

\section{La propriété foncière en Algérie et au Soudan : mise en perspective}

6 L'impact du colonialisme s'est certes traduit par un processus de dépossession foncière. Cependant, comme certains chercheurs tel Babiker l'ont souligné ${ }^{2}$, cela ne fut pas uniformément le cas. Là où la terre pouvait être transférée, les administrations coloniales en ont, en effet, dépossédé les populations indigènes. Mais, là où elle n'était pas recherchée par les colons, la propriété foncière indigène fut souvent renforcée ; l'objectif étant d'empêcher le développement des masses dépossédées mûres pour l'agitation et/ou encore de pourvoir à la subsistance d'une population devenant alors disponible en tant que force de travail. L'Algérie et le Soudan sont des exemples de chacune de ces deux tendances. En Algérie, sous administration française, les terres ont été transférées à grande échelle aux colons européens. Au Soudan, sous administration britannique, la propriété foncière autochtone a été renforcée par le système de la Native Administration.

7 Ces considérations générales doivent néanmoins être nuancées. En Algérie, les politiques sur les terres furent spécifiques selon les régions : les terres sahariennes et de Kabylie semblent avoir moins pâti des mesures de dépossessions foncières. Au Soudan, l'interprétation du régime de propriété collective (communal land tenure) de la part du gouvernement colonial fut en fait assez différente de ce qui prévalait auparavant. Il appartient, en outre, au gouvernement postcolonial d'avoir introduit les mesures de propriété foncière ouvrant la porte aux investisseurs privés. Cela s'est 
traduit par un régime foncier décrit comme «chaotique » et identifié comme l'un des facteurs majeurs des conflits en cours dans ce pays.

Dans les régions du Nord de l'Algérie, jugées attractives pour la colonisation, l'administration coloniale a bel et bien procédé à la dépossession des indigènes au profit des colons européens: quelque 1700000 hectares, en 1900 , et près de 2700000 hectares, en 1940, représentant 35 à $40 \%$ des terres arables de l'Algérie, furent ainsi accaparés par les colons et les grandes entreprises européennes ${ }^{3}$. Ce processus de dépossession fut facilité par la lecture faite des régimes de propriété foncière préexistants. Les terres possédées non pas au titre de la propriété privée ( $m a ̂ l)$ mais en tant que waqf ou habûs (terminologie en cours en Afrique du Nord) - c'est-àdire en tant que biens religieux inaliénables - furent ainsi déclarées aliénables par les autorités coloniales et leur vente aux Européens fut fortement encouragée ${ }^{4}$. Le sujet de la dépossession foncière a attiré l'attention de nombreux chercheurs ${ }^{5}$ dont les travaux sont passés en revue dans deux études portant sur des régions parmi les moins touchées par ces changements : les régions sahariennes et la Kabylie ${ }^{6}$. Il en ressort une image plus nuancée des mutations de la propriété foncière en Algérie durant la période coloniale. Ben Hounet met en évidence comment la dépossession des terres, le discrédit des structures traditionnelles de l'autorité et l'imposition de nouvelles normes culturelles sur les populations autochtones des régions arables du Nord, ont contribué à l'élimination des structures sociales tribales. En revanche, dans les régions sahariennes, la dépossession des terres n'a pas eu lieu et on observe une réification, durant cette période, des structures tribales dans les Territoires du Sud (sous administration militaire $)^{7}$. Scheele souligne que l'administration coloniale marquait peu d'intérêt pour les terres en Kabylie qu'elle considérait comme à la fois distantes et peu fertiles. Elles furent donc négligées dans les premiers temps de la dépossession foncière et ce, jusqu'au soulèvement de 1871 , quand un cinquième des terres de nombreux villages fut confisqué en guise de punition collective. Ce transfert des terres au détriment des villages kabyles et des conseils villageois qui les supervisaient est l'un des éléments que questionne Scheele; son enquête ethnographique à propos de la propriété foncière dans un village montre que les limites des terres du village n'ont pas changé dans les registres ou les témoignages oraux, contrairement aux titres de propriété. Ceci s'explique par la transformation de terres habûs en propriétés privées de manière à éviter la prédation coloniale, l'héritage, les ventes au sein du village et les confiscations qui ont été dans certains cas suivies par une convention collective de rachat des terres. Dans ce contexte, les changements successifs de propriété ont compliqué les répertoires de légitimité des plaintes à propos des propriétés foncières ${ }^{8}$.

$9 \mathrm{Au}$ Soudan, sous les Britanniques, l'administration coloniale a été soucieuse de préserver la gestion indigène de l'accès au foncier, ce qui signifie, entre autres choses, que le règlement des conflits ne relevait pas des compétences des officiers et administrateurs britanniques ${ }^{9}$. Le système de la Native Administration préserva le principe du dâr qui, dans le contexte pastoral soudanais, définissait le "territoire tribal », les droits d'accès à la terre relevant du groupe tribal éponyme.

Babiker montre comment les droits de propriété relatifs au territoire tribal furent néanmoins modifiés durant cette période coloniale. Auparavant, les membres d'un dâr avaient accès à l'ensemble du territoire, y compris ses ressources comme la terre, l'eau et les arbres -ces derniers devinrent importants durant la période coloniale en raison du commerce de la gomme. En contraste, sous le régime de la Native Administration, 
l'accès aux arbres fut restreint à certaines espèces dans des sections définies du dâr. Cela conduisit à une escalade des conflits fonciers, les populations indigènes faisant valoir leurs droits sur davantage d'arbres à mesure que le prix de la gomme augmentait. Les conflits liés au foncier ont continué après l'indépendance.

11 En 1970, deux nouvelles lois contribuèrent à saper le régime du dâr et la notion de terre collective. L'Unregistered Land Act eut pour effet de convertir les terres inhabitées, non cultivées, en propriété de l'État et la Native Administration Abolition Act eut pour résultat de démunir les leaders politiques des groupes locaux de leur autorité concernant les ressources foncières. L'ouvrage édité par Casciarri et Ahmed retrace les effets de ces réformes (et d'autres) sur les populations de pasteurs dont les transhumances reposaient sur les pâturages collectifs - officiellement " non enregistrés ", sans titre de propriété ${ }^{10}$. L'ouvrage montre les collusions entre les mesures politiques relatives au foncier postérieures à l'indépendance et les tendances du marché qui favorisent l'agriculture irriguée et mécanisée sur de vastes échelles, entravant ainsi l'accès des pasteurs à la terre et à l'eau. Casciarri s'intéresse également à la manière dont les pasteurs résistent aux processus de marchandisation induits par les politiques foncières grâce, entre autres, à la vigueur de leurs institutions sociales, tel le travail en commun.

12 Cette mise en perspective permet de situer certains des enjeux actuels des processus d'appropriation foncière. La succession des lois - qu'elles aient eu pour objectif la dépossession ou le renforcement de la propriété des populations autochtones - a fait de la propriété foncière un objet juridiquement complexe, appelant également divers discours de légitimité quant à la reconnaissance des titres et des usages. On esquissera ici certaines des tendances actuelles au niveau des textes de loi, des règles et des pratiques, et des conflits qui leur sont inhérents.

\section{L'accession à la propriété foncière agricole (APFA) en Algérie}

13 La question de l'appropriation du foncier, plus spécifiquement agricole, est un sujet d'actualité en Algérie et ce, en raison de l'adoption par l'Assemblée populaire nationale, durant l'été 2010, d'une loi modifiant les conditions d'exploitation des terres agricoles $^{11}$. Ce texte réserve de manière pérenne à l'État la propriété de terres qui, certes, continuent à relever de son domaine, mais que les exploitants sont à présent autorisés à mettre en valeur par voie de concession. Ceci attise la crainte d'un processus de land-grabbing (accaparement de terres agricoles par des sociétés ou des États étrangers) et les inquiétudes de l'Union nationale des paysans algériens (UNPA). Ainsi, selon le quotidien Le Jeune Indépendant: «Le ministre de l'Agriculture et du Développement rural, Rachid Benaissa, (serait) en train d'examiner une série de candidatures étrangères en vue de l'exploitation de terres agricoles dans le pays. Depuis 2006, les opérateurs étrangers (seraient) autorisés à investir dans [...] les zones sahariennes, steppiques et montagneuses. $»^{12}$

14 Cette loi sur les processus de concession du foncier agricole, dont les effets ne peuvent encore être analysés, se distingue de la loi n ${ }^{\circ} 83-18$, du 13 août 1983 , relative à l'accession à la propriété foncière agricole (APFA), entrée en vigueur en 1984. Celle-ci porte sur des terres relevant du domaine de l'État situées en zone saharienne ou 
présentant des caractéristiques similaires - cas des régions steppiques - ainsi que sur les autres terres relevant du domaine public et susceptibles d'être utilisées, après mise en valeur, pour l'agriculture. Toute personne physique jouissant de ses droits civiques ou toute personne morale de statut coopératif, de nationalité algérienne peut, dans le cadre de cette loi, acquérir des terres agricoles ou à vocation agricole, dans les zones concernées, au prix du dinar symbolique et à condition de la mettre en valeur par ses propres moyens; le transfert de propriété public/privé étant assorti d'une condition résolutoire de cinq ans (sauf cas de force majeure) pour la mise en valeur de la terre, selon un programme élaboré par l'acquéreur et avalisé par l'administration.

La loi relative à l'APFA reprend le principe, que l'on retrouve dans le droit musulman et dans d'autres traditions juridiques, de la vivification (ihyâ) de la terre comme moyen d'appropriation ${ }^{13}$. Ainsi, quiconque vivifie une terre en la cultivant, en la restaurant ou en la rendant utile d'une autre manière, en acquiert la propriété. En 2002, sur l'ensemble de la wilaya de Naama, région steppique et pastorale où nous avons mené nos recherches, 13762,4 hectares de terre étaient attribués au titre de l'APFA et concernaient 4920 bénéficiaires.

Le développement de l'APFA, dans cette zone pastorale, ne se fit toutefois pas au détriment du pastoralisme. De nombreux pasteurs obtinrent une terre dans ce cadre et développèrent simultanément les activités pastorales et agricoles. Ces dernières, souvent complémentaires, favorisaient l'aisance des éleveurs et leur indépendance alimentaire. Les terres obtenues dans le cadre de l'APFA étaient souvent situées sur le territoire des demandeurs et à proximité de l'espace de transhumance du bétail.

Lors de notre dernier séjour, en octobre 2010, dans la wilaya de Naama, le programme de l'APFA avait été suspendu en raison de la nouvelle loi évoquée plus haut mais, également et surtout, en raison de conflits successifs générés par l'attribution (ou non) de parcelles agricoles au titre de l'APFA et par la question de l'effectivité des mises en valeur des terres, au terme des cinq années prévues par la loi. Le droit d'accession à la propriété foncière agricole se heurte, en effet, en zone pastorale, à plusieurs difficultés pratiques: les contraintes bureaucratiques, souvent arbitraires, qui freinent, voire empêchent l'accession à la propriété foncière de certains demandeurs au bénéfice d'autres requérants ; les conflits s'agissant des règles d'usage et des droits collectifs des zones de parcours des pasteurs; les contraintes économiques qui ne permettent pas l'investissement et donc la mise en valeur des terres, etc. Se superpose également, explicitement ou non, le droit de l'APFA et les pratiques administratives d'attribution de propriétés foncières agricoles et de suivi de la mise en valeur ; les usages coutumiers ('urfiyya) s'agissant de l'exploitation des terres de la part des pasteurs/éleveurs et les droits sur la terre ; le droit musulman concernant le principe de la vivification. De fait, l'accession à la propriété foncière semble moins difficile lorsque le requérant établit une demande sur une parcelle située "sur les terres » de son clan ('aqîla) et s'il dispose des moyens nécessaires à sa mise en valeur (capitaux économiques et sociaux). Elle semble plus compliquée lorsque le requérant n'appartient pas au clan qui fait traditionnellement usage du territoire sur lequel se trouve la parcelle, bien que la loi et le principe de vivification des terres puissent être avancés. L'analyse des conflits et des mécanismes judiciaires et parajudiciaires se révèle donc fort utile à la compréhension des droits réels de propriété et d'appropriation, des logiques d'appropriation en contexte, et des argumentaires et recours juridiques mobilisés à ces fins. Notons que l'article 15 de la loi de l'APFA stipule que la « condition résolutoire (au terme des cinq 
années) invoquée par l'autorité administrative compétente est dans tous les cas appréciée par voie judiciaire ».

\section{De la propriété foncière au Soudan}

L'analyse des conflits fonciers, ruraux mais aussi urbains, est cruciale pour la compréhension d'une grande part des problèmes politiques, locaux et régionaux qui touchent la société soudanaise. Ces conflits sont évidemment liés aux divers modes d'accès à la terre, donc aux différentes formes de propriété et aux divers modes de tenure du sol - agricole, pastoral, forestier -, de construction d'habitat ainsi que d'implantation d'infrastructures et d'équipements industriels ou publics. Ces usages varient selon les régions et fractions du territoire, urbaines ou rurales et, parmi cellesci, selon les grandes zones écologiques. Pour ne prendre que l'exemple des usages agricoles, l'agriculture soudanaise est soit irriguée soit pluviale. Le secteur d'agriculture irriguée (AI), le long du Nil et de ses affluents, comprend d'une part un sous-secteur traditionnel (AIT) de petite et moyenne paysannerie, cultivant en irrigation fluviale à l'aide de méthodes traditionnelles d'exhaure ou de pompes individuelles et, d'autre part, un sous-secteur moderne (AIM) de grands périmètres d'État irrigués par canaux ou par pompes industrielles. L'agriculture pluviale (AP), quant à elle, regroupe d'une part le sous-secteur moderne (APM) des grandes exploitations pluviales mécanisées, implantées sur une ceinture sud-sahélienne courant de l'Est du Soudan au Sud-Darfour et, d'autre part, l'immense diversité régionale des agricultures paysannes pluviales dites «traditionnelles» (APT), réparties sur l'ensemble des zones sahéliennes, de la frontière éthiopienne au Darfour. Ces immenses zones sahéliennes sont également le domaine d'intenses activités pastorales menées par des populations sédentaires (paysannes en général), transhumantes saisonnières ou réellement nomades, ces deux dernières étant spécialisées dans l'élevage.

Les modes d'accès à la terre varient selon ces quatre sous-secteurs agricoles et l'on peut avancer très grossièrement que, depuis l'Unregistered Land Act de 1970, les terres qui, avant cette date, n'avaient pas été enregistrées comme "privées " (selon la Land Registration Ordinance de 1925) et donc réputées propriété éminente de l'État représentent la quasi-totalité des terres sahéliennes d'AP: les terres des grandes exploitations pluviales mécanisées (sous-secteur APM) sont données à bail par l'État à de gros exploitants et relèvent donc du droit foncier " étatique »; les terres du soussecteur APT sont aussi en général propriété de l'État, qui en concède la jouissance collective aux communautés villageoises et «tribales ", aux membres desquelles leurs autorités «traditionnelles» en (ré)attribuent périodiquement l'usufruit, selon des droits dits « coutumiers » extrêmement divers en fonction des régions, des ethnies et des entités tribales (notons une tendance à l'appropriation "privative », tolérée par la coutume, des terres maraîchères riveraines des wadi). Entre ces communautés villageoises et les groupes pastoraux transhumants ou nomades, se négocient les droits coutumiers saisonniers de passage et de pacage. Les terres du sous-secteur AIM (grands périmètres irrigués) sont aussi propriété de l'État qui les a concédées aux Bords et Corporations publiques qui les attribuent par lots de tailles variables à des tenants titulaires, censés les travailler par eux-mêmes et pouvant en hériter, mais les donnant souvent à bail, en fermage ou métayage. Enfin, le sous-secteur AIT, riverain du Nil, en particulier en aval de Khartoum, est par excellence le domaine de la petite propriété 
privée. Notons que si les droits coutumiers et positifs-étatiques interviennent principalement dans le domaine foncier, il existe, au sein même de ces droits, des éléments de "droit musulman ", en particulier en matière d'héritage. Ces éléments, souvent réinterprétés et « confrontés » aux droits coutumiers, interviennent largement dans la résolution des conflits fonciers, dont on imagine sans peine la fréquence, vu la diversité et l'enchevêtrement local des modes d'accès au sol, sa dévolution ne consistant que peu en héritage et vente de biens fonciers privés et, plus souvent, en cession et attribution de "droits» partiels sur la terre par divers acteurs institutionnels ou collectifs à d'autres types d'acteurs institutionnels, collectifs ou individuels.

20 Au regard des modalités des "variables contextuelles » succinctement énoncées cidessus et entrant dans la description et le traitement des conflits, les zones rurales et urbaines du Nord-Soudan offrent une extraordinaire diversité à chaque échelle - les villages d'une même petite zone offrant certaines modalités communes à la zone et d'autres spécifiques au village, structure que l'on retrouve à l'échelle des zones rurales d'une même région. Ceci sans parler bien sûr de la spécificité de chaque conflit-litige analysé et de son déroulement.

21 De fait, les transformations les plus récentes de la société soudanaise, très diverses selon les contextes régionaux, interdisent la saisie d'un hypothétique contexte général d'analyse (social, politique, économique, culturel) qui constituerait l'arrière-plan des pratiques étudiées. Les reconfigurations multiples du social et les formes de transition que ce dernier revêt peuvent être résumées ainsi :

22 - l'urbanisation accélérée, la croissance des villes et de leurs périphéries, l'exode rural et la sédentarisation des nomades, la croissance des flux de populations déplacées à la suite de conflits armés, la recomposition des classes et l'émergence d'une nouvelle bourgeoisie ; on assiste donc à la création d'espaces, de groupes sociaux et de pratiques sociales «hybrides» qui rendent difficiles l'application des catégories nettement contrastées « urbain » et « rural»;

23 - l'accélération des phénomènes de land grabbing, de restructuration des modes d'accès aux ressources et de leur exploitation, d'expropriation foncière et de privatisation, qui mènent à des changements de statut juridique des biens et ressources et au bouleversement des catégories locales permettant de penser leurs modes d'appropriation et leurs usages ;

24 - la multiplication et la diversification des acteurs impliqués dans les pratiques juridiques et l'impossibilité de trancher entre des populations et les pratiques qui relèveraient plutôt de l'ordre du rural-traditionnel-tribal-«droit coutumier » et d'autres qui, par contraste, seraient à inscrire dans l'ordre de l'urbain-moderneétatique-« droit positif ou islamique ».

On donnera quelques exemples d'études de cas qui aident à illustrer la variété et la complexité des contextes des pratiques juridiques que l'on essaiera de saisir :

26 Cas 1: Deux groupes tribaux/pastoraux en conflit, avec l'État et son droit comme garants. Le territoire aride périphérique (Khanjar) occupé par une population d'ancienne tradition pastorale - largement sédentarisée - acquiert une nouvelle centralité après l'expansion de la capitale et l'installation de la deuxième raffinerie du pays par les Chinois, en 2001. Les terres gérées depuis longtemps, sans conflits majeurs, comme champs d'agriculture pluviale deviennent un nouvel espace de compétition pour les bénéfices possibles que 
leur accaparement dans cet espace récemment «dé-ruralisé » laisse envisager. Deux tribus d'origine nomade, les Ahamda et les Kababish, s'affrontent ainsi pour revendiquer leurs droits exclusifs. Le conflit est porté devant les tribunaux mais les chefferies tribales sont convoquées et leurs témoignages (selon des critères " coutumiers» d'appropriation des terres collectives) sont sollicités par la justice étatique pour résoudre le conflit. L'issue finale déclenche la création d'une instance locale de partage des terres collectives au sein du groupe qui sort gagnant du conflit.

Cas 2: Un groupe pastoral sédentarisé versus un groupe migrant, des "comités populaires" étatiques imprégnés de logiques "tribales». Dans une zone périurbaine de l'est de la capitale (Babiker), les primo-arrivés (non autochtones) Batahin, sédentarisés à la suite de la famine de 1949-1950, se trouvent confrontés à l'expansion et à la régularisation de squatter areas, peuplées surtout de Sudistes et de personnes déplacées, et à la croissance de la ville, ce qui engendre une redéfinition constante des formes d'accès à la terre. Les négociations se font au cas par cas entre les groupes ou les familles récemment installés et ces primo-arrivés reconnus localement (mais pas officiellement par l'État) comme ayants droit principaux. À Bauga, un groupe originaire du nord de la vallée du Nil récemment arrivé dans la localité élabore ses stratégies pour "acheter » la terre où s'installer: le conflit/négociation entre ces "étrangers» et les anciens nomades sédentarisés, ayants droit principaux, se double des dissensions internes au groupe Batahin (entre ceux qui défendent l'inaliénabilité des terres tribales et ceux qui profitent de la «vente » de ces terres) et s'appuie sur des relectures instrumentales du rôle des Comités populaires (création étatique datant de 1989) dans l'aliénation des terres.

Cas 3: Un groupe tribal «dé-tribalisé » de la capitale en conflit avec l'État au sujet de la vente de terres aux classes moyennes récemment installées. À Salha, situé au sud de la périphérie d'Omdurman (partie occidentale de la capitale), l'expansion du centre et l'arrivée massive de nouvelles populations dans les marges, doublées de nouveaux projets d'aménagement urbain, font ressurgir dans l'espace urbain les revendications d'anciens groupes tribaux. Le groupe Jamuya, dominant historiquement la zone et " dé-tribalisé » par son insertion ancienne dans la ville, met en place des stratégies nouvelles, parallèles à celles de l'État et s'appuyant sur une relégitimation tribale, pour contrôler la distribution et la vente des terres à des membres de classes moyennes cherchant à s'installer dans la zone. Le statut qu'ils tentent de conquérir dans ces transactions foncières urbaines en négociant avec ces derniers (très différents des populations marginales habituellement intéressées par l'installation dans les aires périphériques) est en opposition avec l'État et son droit.

\section{Conclusion}

L'observation de ces diverses configurations et de leurs dynamiques spécifiques permet de saisir, ici autour d'un objet commun (les modes d'accès à la terre), le jeu des différents acteurs (État, individus, collectivités lignagères ou «tribales »), leurs différents référents juridiques (droit coutumier, droit islamique, droit civil), les institutions impliquées dans l'arène de la négociation pour l'accès à la terre et la résolution éventuelle du conflit. Si la dimension conflictuelle n'est pas notre point exclusif de départ, la connaissance du terrain nous permet de penser que les conflits autour de la terre, dans ses différents usages (comme terre agricole et ressource 
«naturelle ", comme espace constructible, voire comme pur support de spéculation), constituent un objet d'observation privilégié pour comprendre les formes et les pratiques juridiques qui prévalent dans des contextes musulmans variés.

\section{BIBLIOGRAPHIE}

BABIKER M., 2009, « Pastoral Land Rights and Peace-Building in North Kordofan: Policy and Legislative Challenges », Nomadic Peoples, vol. 13, $n^{\circ} 1$, special issue, (« Pastoralists under pressure in present-day Sudan », B. Casciarri and A. G. Ahmed eds) : 134-153.

BÉDRANI S., 2001, Les contraintes au développement des zones steppiques et la mise en valeur par les concessions, Alger, ministère de l'Agriculture, $30 \mathrm{p}$.

BEN HOUNET Y., 2009, L'Algérie des tribus : le fait tribal dans le Haut Sud-Ouest contemporain, Paris, L'Harmattan.

BENNOUNE M., 1986, El-Akbia : un siècle d'histoire algérienne, 1857-1975, Alger, Office des publications universitaires.

BOURDIEU P. et A. SAYAD, 1964, Le déracinement : la crise de l'agriculture traditionnelle en Algérie, Paris, Éditions de Minuit.

CASCIARRI B., 2009, « Between market logic and communal practices: pastoral nomad groups and globalization in contemporary Sudan (case studies from central and western Sudan) ", Nomadic Peoples, vol. 13, n $1: 69-91$.

CASCIARRI B. and A. G. AHMED, 2009, « Pastoralists under pressure in present-day Sudan: an introduction ", Nomadic Peoples, vol. 13, $\mathrm{n}^{\circ} 1: 10-22$.

DENOIX S., 1996, «Introduction : Formes juridiques, enjeux sociaux et stratégies foncières ", Revue du monde musulman et de la Méditerranée, $\mathrm{n}^{\circ}$ 79-80 : 9-22.

LAPIDUS I. M., 2002, A history of Islamic societies, Cambridge, Cambridge University Press.

LAUNAY M., 1963, Paysans algériens : la terre, la vigne et les hommes, Paris, Seuil.

NOUSCHI A, 1961, Enquête sur le niveau de vie des populations rurales constantinoises, de la conquête jusqu'en 1919: essai d'histoire économique et sociale, Paris, Presses universitaires de France, LXXIII-769 p ; université de Tunis, publication de la Faculté des lettres,4c série, vol. 3.

POWERS D. S., 2002, The Encyclopaedia of Islam, H. A. R. GIBB, T. H. BIANQUIS, C. E. BOSWORTH, E. VAN DONZEL and W. P. HEINRICHS, Leiden, Brill, XI, Wakf [in North Africa to 1914] : 69-73.

SCHEELE J., 2009, Village matters: knowledge, politics \& community in Kabylia, Algeria, Woodbridge, Suffolk, James Currey : XII.

\section{ANNEXES}




\section{Propriété, contrat et droit : approche théorique}

Durkheim fait de la protection de « la propriété de la personne humaine » la seconde règle de la morale humaine (Leçons de sociologie, p. 152). Il situe la question du droit contractuel dans le fil de cette interrogation, c'est-à-dire dans la perspective des modes d'acquisition et de cession de la propriété, dont l'héritage est un des cas de figure. Contrairement à la conception classique, pour laquelle la propriété est liée au travail et se présente comme un droit naturel - Stuart Mill affirme, par exemple, que « la propriété n'implique rien d'autre que le droit de chacun sur ses talents personnels, sur ce qu'il peut produire en les appliquant »-, Durkheim fait remarquer que la propriété et partant, son transfert mais aussi les liens de parenté qui le justifient - est un fait social. Mais, un fait social peut finir par s'imposer comme la «nature des choses » par sa seule existence, « un agencement tel de faits et de circonstances qu'une fois son existence reconnue, il paraît inexorable » (Foriers, 1982).

Kant considère la propriété comme un lien intellectuel, puisqu'il associe une personne et une chose indépendamment de toute nécessité de lien physique. Cette relation suppose en même temps la réciprocité, dès lors que, pour pouvoir faire admettre à autrui son droit sur quelque chose qu'il ne détient pas physiquement, ego doit luimême admettre le droit équivalent d'autrui sur des biens différents mais semblables. Et, pour que cette réciprocité soit établie, « il faut que les choses soient originellement possédées par une collectivité ». Durkheim, en partant de la conception kantienne, cherche une définition du droit de propriété qui soit abstraite des modalités particulières qu'il a pu prendre dans les différents temps et lieux. Le premier élément qu'il identifie est l'existence d'une chose appropriable, dont la définition, bien entendu, varie d'une population à l'autre, tout comme varie la définition de celui qui peut posséder. Le second élément tient au rapport entre le sujet possédant et la chose possédée, que Durkheim spécifie comme étant la jouissance exclusive du bien quand bien même la jouissance effective serait différée dans le temps, comme dans le cas de l'usufruit. Cela permet d'interdire à autrui d'en user. Cette question de la relation est aussi au cœur de la définition de la propriété que propose Lévi-Strauss. Il ne s'agit plus ici d'une relation objective entre le sujet et l'objet, mais d'une relation entre personnes, une relation à autrui et à la structure sociale (Les Structures, p. 100), dont on peut toutefois dire qu'elle est « médiatisée » par le bien possédé. Il faut également mentionner l'approche matérialiste, comme celle de Godelier, qui considère que les formes de propriété représentent en réalité des rapports de production. Le droit est ici considéré comme une superstructure dissimulant le contenu réel de ces rapports de production, parfois même en décalage par rapport à ceux-ci (le droit n'est plus alors qu'une fiction).

L'étude de la propriété ne se résume bien entendu pas à Kant, Durkheim, Lévi-Strauss et Godelier. Il nous importe ici de souligner que tous ces auteurs se sont lancés dans la quête d'une ontologie de la propriété, c'est-à-dire une recherche de ces propriétés essentielles qui en fondent l'« être ». D'une certaine façon, cependant, cette ontologie se réduit souvent à une métaphysique, en ce sens que la nature essentielle de cet « être » les préoccupe davantage que les conditions de son déploiement. Pour notre part, nous proposons, à partir de deux contextes très spécifiques, de suspendre cette question de l'essence du droit de propriété pour en explorer la praxéologie ; autrement dit, de mener la description ethnographique des pratiques qui lui sont liées. Celle-ci 
passe par l'identification des modes d'appropriation d'un bien, des moyens permettant d'attester d'un droit de propriété, des recours ouverts pour le faire valoir, des façons de raisonner dans ces instances dès lors qu'il faut établir des causes, des effets ou des relations, de la part jouée par la parole ou l'écrit, des conceptions « en acte » de notions importantes telles que les distinctions personne-bien, meuble-immeuble, propriétépossession, propriété-jouissance, etc.

Longtemps, le contrat a été considéré comme un fait propre aux sociétés modernes. Henri Maine (Ancient Law) est connu pour son affirmation que, dans les sociétés traditionnelles, le statut social des personnes génère les droits, obligations et responsabilités plus que les volontés individuelles. Dans la ligne de Maine, Durkheim associe sa célèbre distinction entre les solidarités mécanique et organique à la distinction entre statut (mécanique) et contrat (organique) et entre droits répressif (mécanique et statutaire) et restitutif (organique et contractuel), tout en se gardant d'établir des oppositions trop rigides et en traitant la question en termes tendanciels. La thèse de Marcel Mauss sur le don et le contre-don, dans le prolongement des travaux de Durkheim, a tendu à affranchir la sociologie et l'anthropologie du contrat de la perspective individualiste (e.g. Malinowski) pour l'inscrire dans le contexte plus large d'un tissu social nouant des personnes, des objets et des relations.

De nombreuses études de facture ethnographique ont mis à mal la thèse de Maine. Le réaliste américain Hoebel a ainsi approfondi les intuitions de Durkheim sur la nature non exclusive des relations statutaires et contractuelles. Un théoricien du pluralisme juridique, Leopold Pospisil, a par ailleurs montré que les relations contractuelles pouvaient également précéder les relations statutaires. Toutefois le caractère évolutionniste de la thèse a concentré la critique, alors que la distinction entre droit statutaire et droit contractuel continue à structurer les approches. Étienne Le Roy, par exemple, distingue les types d'accord en fonction des structures et des logiques sociétaires. Il établit ainsi un continuum allant des structures élémentaires, à la logique communautariste et au système d'échange fondé sur le don, aux structures complexes, à la logique individualiste. Ces structures sont marquées par la prédominance du contrat. Ce type d'associations est cependant contesté par un pan important de l'anthropologie (Allott, Gluckman, Sahlins), qui considère que les relations contractuelles ne peuvent se développer qu'entre des personnes entretenant des relations sociales minimales.

La caractéristique de l'anthropologie du contrat est bien sa propension à vouloir modéliser les transactions et à les inscrire dans une séquence déterministe - quand bien même cette séquence pourrait parfois être inversée. L'ethnographie des relations contractuelles apparaît en fin de compte comme le parent pauvre de cette démarche : on a étudié le contrat davantage comme ressource explicative de la nature des sociétés qu'en lui-même et pour lui-même, comme instrument de formalisation de transactions, comme appui documentaire, comme moyen probatoire, comme source de saisine des instances de règlement de litiges, bref, comme objet qui s'inscrit dans un ensemble de pratiques dont seule la description en contexte et en action nous permet de rendre compte et d'expliquer. Autrement dit, nous restons confrontés à ce « quelque chose qui manque » des études socio-anthropologiques, à savoir la description ethnographique de ce qui fait l'héritage et la transmission du patrimoine par voie de succession, non pas au niveau de structures qui échapperaient pour une large part aux gens concernés, 
mais dans des pratiques qu'ils connaissent ou reconnaissent, des pratiques qu'ils tiennent pour normale et qui dès lors entrent dans une routine.

L'anthropologie du droit, dans son souci de résoudre les problèmes liés à l'impossibilité de donner une définition incontestable au terme de droit, s'est depuis longtemps tournée vers l'étude des cas litigieux et des processus de règlement des conflits. La méthode des trouble-cases du réalisme américain, qui remonte à The Cheyenne Way de Llewellyn et Hoebel, a fondé nombre d'études du droit dans les sociétés sans État. Plus récemment, l'étude du règlement du conflit s'est transformée en étude du processus conflictuel, sous l'impulsion, entre autres, de l'anthropologue Laura Nader, pour finir par devenir, avec Sally Falk Moore, l'étude du droit en tant que processus. Dans cette évolution, on voit que la restitution de cas problématiques tels que s'en souviennent des informateurs ne constitue pas le seul matériau de l'anthropologie, mais aussi l'ethnographie du déroulement des litiges et de leur règlement, dans une dimension à la fois processuelle et procédurale. Le livre de Gluckman, The Judicial Process among the Barotse (1955), est l'ouvrage à la fois fondateur et inégalé de cette tendance anthropologique. L'un des intérêts principaux de la démarche réside dans l'attention portée aux modes de raisonnement propres aux personnes impliquées dans le processus, qui ne sont pas abstraits du contexte de leur déploiement, mais au contraire articulés systématiquement à celui-ci.

\section{NOTES}

1. Notre contribution s'appuie sur un programme en cours portant sur l'anthropologie du droit dans les sociétés en tout ou en partie musulmanes (programmes Andromaque, ANR Suds II, et Prométhée, ANR FRAL) et, plus précisément, sur la question de la "propriété » et de sa transmission. On se référera au texte en annexe qui présente, de manière synthétique, l'approche théorique de cet article et du programme de recherche sur lequel il s'appuie.

2. Voir M. Babiker, « Land tenure in Kordofan: Conflict between the "communalism" of colonial administrators and the "individualism" of the Hamar ", in E. Stiansen and M. Kevane (eds), Kordofan invaded: peripheral incorporation and social transformation in Islamic Africa 1785-1995, Leiden, E. J. Brill, 1998 : 197-222.

3. Lapidus $2002: 589$.

4. Powers 2002 .

5. Nouschi 1961 ; Launay 1963 ; Bourdieu et Sayard 1964 ; Bennoune 1986.

6. Pour les régions sahariennes, voir Ben Hounet 2009 et pour la Kabylie, Scheele 2009.

7. Ben Hounet $2009: 17-23$ et 23-24.

8. Scheele 2009 : 64-66.

9. M. Babiker, art. cité (1998) et 2009.

10. Casciarri and Ahmed 2009.

11. La loi $n^{\circ} 10-03$ du 15 août 2010 fixant les conditions et les modalités d'exploitation des terres agricoles du domaine privé de l'État a été publiée dans le Journal officiel, $\mathrm{n}^{\circ} 46$, du 18 août 2010.

12. "Ouverture du secteur agro-industriel aux multinationales. Plusieurs groupes mondiaux intéressés ", Le Jeune Indépendant, 16 septembre 2010.

13. Denoix 1996. 


\section{RÉSUMÉS}

Cet article porte sur la question de la « propriété » et de sa transmission. Nous proposons de nous départir d'une vision culturaliste du droit, qui prévaut largement dans les travaux sur les mondes musulmans, au profit d'une approche ethnographique des pratiques d'acquisition et de transmission de la propriété foncière. Nous montrons comment, sur des terrains algériens et soudanais, les acteurs mobilisent des droits locaux et nationaux, voire internationaux, pour faire valoir un titre à la propriété, en particulier sur des biens collectifs ou publics. L'attention est focalisée sur les pratiques actuelles d'appropriation foncière en zone agricole et/ou périurbaine et sur les conflits qui leur sont inhérents. Après avoir succinctement présenté le cadre "praxéologique » de la démarche que nous entreprenons, ainsi que les travaux récents au sujet de la propriété dans ces deux pays, nous décrivons comment opèrent, autour d'un objet commun (les modes d'accès à la terre), le jeu des protagonistes, leurs différents référents juridiques et les institutions impliquées dans l'arène de la négociation pour l'accès à la terre et la résolution éventuelle du conflit.

This article deals with the question of "property" and its transmission. Our approach distances itself from the right-wing culturalist perspectives often adopted in studies of the Muslim world to favour an ethnographical approach examining the ways in which land is acquired and transferred. We show how actors in Algeria and Sudan use local, national and even international laws to claim title to land, especially in cases of collective or public goods. Our focus then shifts to the land appropriation practices currently affecting agricultural and/or peri-urban areas and the conflicts they give rise to. After briefly presenting our "praxelogical" approach and recent work on property in both countries, we describe how protagonists's strategies, different legal referents, and institutions involved in negotiations on land access and conflict resolution work towards a single goal (providing access to land).

\section{INDEX}

Mots-clés : droit, propriété foncière, mondes musulmans, praxéologie

Keywords : law, land ownership, Muslim world, Algeria, Sudan, praxeology

Index géographique : Algérie, Soudan

\section{AUTEURS}

\section{YAZID BEN HOUNET}

Centre Jacques Berque

yazid_benhounet@yahoo.fr

\section{BARBARA CASCIARRI}

Université de Paris-VIII

barbara.casciarri@gmail.com

BAUDOUIN DUPRET

CNRS/CJB (Centre Jean Bérard)

baudouin.dupret@cjb.ma 
FRANÇOIS IRETON

CNRS

iretonf2@live.fr

ALICE WILSON

University of Cambridge

arw42@cam.ac.uk 